\title{
Quigouverne? Le Forum de Davos et le pouvoir informel des clubs d'élites transnationales
}

\author{
JEAN-ChRISTOPHE GRAZ
}

Depuis plus de trente ans, les principaux responsables économiques et politiques de la planète se retrouvent fin janvier à Davos pour la réunion annuelle du Forum économique mondial (WEF selon l'acronyme anglais en vigueur) ${ }^{1}$. Le pouvoir incarné par cette grande messe informelle donne lieu à des analyses qui varient du tout au tout. Dans un éditorial du Monde diplomatique, les termes de «rendez-vous des nouveaux maîtres du monde» et d' "aréopage des élites» sont mobilisés à dessein pour dénoncer le pouvoir illégitime que s'arroge un tel rassemblement ${ }^{2}$. En revanche, pour Georges Soros, le grand financier devenu philanthrope en chef de l'Europe centrale et orientale, le Forum de Davos ne serait rien d'autre qu'une "énorme cocktail party» 3. Ces vues contrastées sur l'influence du WEF à l'échelle de l'économie et de la politique internationale reflètent assez fidèlement les positions de ceux qui les tiennent: les opposants dénonçant son pouvoir et les habitués le minimisant. Du point de vue théorique, l'évidence de ce désaccord renvoie pourtant à une question cruciale du débat sur la mon-

\footnotetext{
* Je remercie le Fonds national suisse de la recherche scientifique (FNS) pour le financement de la recherche «Gouvernance commerciale mondiale: entre pouvoirs publics et autorité privée» dans laquelle cette contribution prend place.

Je remercie aussi les membres de l'Institut universitaire de Hautes Études internationales (HEI) à Genève, du Centre d'Études et de Recherche internationales (CERI) à Paris et du Centre for Global Political Economy de l'Université de Sussex pour leur soutien, leurs précieux conseils et l'environnement fructueux qu'ils m'ont offert durant les différentes étapes de cette recherche.
} Je remercie enfin les nombreuses personnes associées de près ou de loin au WEF qui m'ont donné l'occasion de m'entretenir avec elles.

${ }^{1}$ Cette contribution s'appuie sur un travail d'enquête recourant aux archives officielles et privées accessibles du Forum économique mondial et sur des entretiens semi-directifs avec une quarantaine de personnes associées au Forum du point de vue de l'organisation ou en tant que participants. Cette recherche m'a aussi conduit à dresser un questionnaire adressé électroniquement à 715 personnes qui avaient participé aux éditions 2000 et 2001 du Forum de Davos. Mon initiative a motivé une réaction immédiate du directeur général du Forum, m'enjoignant de cesser immédiatement d'importuner de la sorte les membres du Forum et, vraisemblablement, les dissuadant de donner suite à mon initiative. Cette dernière démarche, le biais dans les adresses disponibles pour l'envoi du questionnaire et son taux de retour insuffisant m'ont conduit à estimer que les exigences méthodologiques d'une analyse quantitative n'étaient, dans de telles conditions, pas remplies.

\footnotetext{
2 Ignacio Ramonet, Le Monde diplomatique, mars 1996, p. 1.

3 Cité in «I, Klaus», Wired Magazine, 7 décembre 1999.
} 
dialisation: comment penser le pouvoir économique et politique à l'échelle mondiale au-delà des États et des opérateurs du marché?

L'emprise accrue des acteurs non étatiques sur la scène mondiale a conduit à appréhender le pouvoir informel comme un enjeu crucial des relations internationales. Un nombre croissant de travaux examinent les nouvelles formes d'action collective et d'autorité internationale qui en découlent. Le domaine d'étude est large: il peut aussi bien se rapporter aux mécanismes de coopération entre entreprises mondialisées qu'aux réseaux d'organisations non gouvernementales, aux think tanks les plus influents qu'aux sociétés de sécurité privées, aux grandes agences de notation des titres de propriété qu'à la mondialisation par le bas des réseaux de migrants nomades ${ }^{4}$. Dans un travail pionnier sur le sujet, Susan Strange reprenait la question classique du "Qui gouverne?»5. Ultérieurement, elle reconnaissait qu'une analyse en profondeur de la question requérait «un examen plus minutieux de l'étendue et des limites de l'autorité non étatique" ${ }^{6}$. Parmi les recherches actuelles, l'objet du pouvoir informel dans les relations internationales, la nature de son influence et sa relation avec l'État restent en effet largement équivoques. Selon Cutler, la localisation de l'autorité sur la scène mondiale renvoie à un espace indéfini «entre l'économique et le politique, les activités privées et publiques et une économie politique locale et globale» ${ }^{7}$. Hibou aborde le même problème en soulignant que «la privatisation correspond moins à une baisse du public au profit du privé qu'à une nouvelle combinaison entre public et privé» ${ }^{8}$.

Les clubs d'élites transnationales tels que le Forum économique mondial constituent un cas exemplaire de cette problématique. Définir leur pouvoir a toujours constitué une difficulté d'envergure du point de vue de la recherche empirique. Leur influence sur telle ou telle question de politique internationale repose sur l'usage de canaux informels et diffus. Ce simple constat a une double portée: méthodologique-

\footnotetext{
4 Voir notamment: John Braithwaite and Peter Drahos, Global Business Regulation, Cambridge Cambridge University Press, 2000; Claire Cutler et al. (eds), Private Authority and International Affairs, New York: SUNY Press, 1999; Rodney Bruce Hall and Thomas J. Bierstecker (eds), The Emergence of Private Authority in Global Governance, Cambridge: Cambridge University Press, 2002; Béatrice Hibou (dir.), La privatisation des États, Paris: Karthala, 1999; Richard Higgott et al. (eds),
}

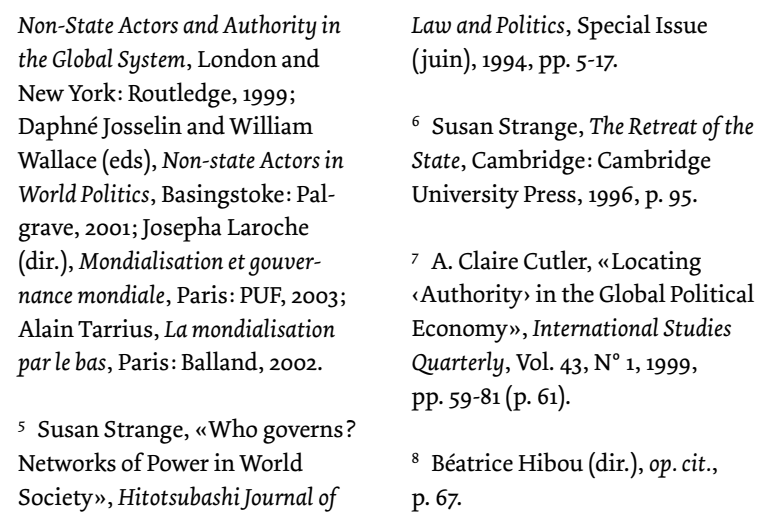

Law and Politics, Special Issue (juin), 1994, pp. 5-17. 
ment, il rend intenables les conclusions qui ressortiraient d'une enquête fondée sur une recherche de la preuve; et théoriquement, il exclut le recours à une approche béhavioriste du pouvoir focalisée sur les pratiques décisionnelles par lesquelles l'aptitude à mobiliser des ressources se transforme en résultats tangibles. Il paraît en effet illusoire de retracer les liens entre une position défendue dans le confinement informel d'un club fréquenté par des élites et sa translation finale dans le cadre formel d'une décision politique. La difficulté s'estompe, en revanche, si l'on adhère à une conception plus structurelle du pouvoir, focalisée sur l'environnement dans lequel les interactions stratégiques des agents prennent place ${ }^{9}$. De ce point de vue, les clubs d'élites transnationales se situent au cœur de l'organisation politique du capitalisme à l'échelle mondiale. Ils contribuent à identifier des problèmes, à leur opposer un langage commun, à clarifier les lignes de forces dans lesquelles ils se situent - et plus prosaïquement, ils constituent un lieu privilégié pour négocier des dossiers de toutes sortes. La plupart des études n'en restent pas moins équivoques sur le processus de socialisation par lequel se développe concrètement leur emprise sur la société et n'envisagent pas les limites dans lesquelles il faut circonscrire leur influence ${ }^{10}$. Il est caractéristique à cet égard que le cas particulier du Forum de Davos soit si souvent mentionné sans qu'aucune recherche d'envergure n'ait jamais été engagée à son sujet ${ }^{11}$.

La présente contribution soutient la thèse que le pouvoir informel des clubs d'élites transnationales est intrinsèquement limité par leur principe d'organisation et les

9 De façon générale, le pouvoir structurel se réfère aux structures matérielles et discursives qui affectent de façon intentionnelle et inintentionnelle le champ du possible d'un ensemble d'acteurs sociaux. Voir en particulier: Stefano Guzzin, «The use and misuse of power analysis in international theory", in Ronen Palan (ed.), Global Political Economy: Contemporary Theories, London and New York: Routledge, 2000, pp. 53-66.

10 Stephen Gill, American Hegemony and the Trilateral

Commission, Cambridge: Cambridge University Press, 1990; Kees van der Pijl, The Making of the Atlantic Ruling Class, London: Verso, 1984 et Transnational Classes and International Relations, Lon don: Routledge, 1998; Bastiaan van Apeldoorn, Transnational Capitalism and the Struggle for European Integration, London: Routledge, 2002; Dominic Kelly, «Beyond Diplomacy: The International Chamber of Commerce and Global Governance», CSGR Working Paper, Vol. 74/01, 2001; Leslie Sklair, The Transnational Capitalist Class, Oxford: Blackwell, 2001.

${ }^{11}$ Le seul article scientifique sur le WEF est consacré à une problématique pédagogique et repose sur des sources secondaires et les documents en ligne du site internet du Forum. Il défend la thèse que le WEF constitue un cas multifonctionnel exemplaire pour comparer les théories concurrentes du champ d'étude de l'économie politique internationale. Voir: Geoffrey Allen Pigman, «A Multifunctional Case Study for
Teaching International Political Economy: The World Economic Forum as Shar-pei or Wolf in Sheep's Clothing?", International Studies Perspectives, Vol. 3, 2002, pp. 291-309. Le manuel Politique internationale de Josepha Laroche (Paris: LGDJ, 200o, pp. 187-192) consacre une demi-douzaine de pages au WEF en tant qu'espace de diplomatie informelle jouant le rôle de creuset de la politique internationale; l'étendue et les limites du pouvoir informel véhiculé par ce genre d'entreprise ne fait pas l'objet d'une analyse détaillée. Enfin, il existe un petit essai du rédacteur en chef de la revue américaine Harper's Magazine relatant avec cynisme son expérience davosienne à la fin des années 9o: Lewis Lapham, $\mathrm{La}$ montagne des vanités, Paris: Maisonneuve \& Larose, 2000. 
modalités concrètes de leur pratique. Cet argument s'appuie sur deux hypothèses conjointes: la première consiste à supposer que de tels groupes rassemblent des acteurs étant de facto en position de pouvoir; au lieu de chercher à prouver leur influence, il s'agit dès lors de la prendre pour telle et en évaluer les limites. La seconde pose que le principe d'exclusion inhérent à une organisation élitaire explique à la fois la force et la faiblesse de son pouvoir informel. Cette démarche, qui s'apparente à une herméneutique des limites, emprunte à Georges Sorel la notion de mythe social. Il s'agit d'appréhender l'idée selon laquelle le sentiment d'appartenir à une communauté exclusive partageant une responsabilité particulière envers l'avenir du monde peut susciter une "passion collective» ayant, certes, des répercussions tangibles sur la réalité, mais dont la portée reste limitée en raison de l'absence de relations formelles avec les institutions politiques de la société1 ${ }^{12}$. De ce point de vue, la pratique des clubs d'élites transnationales repose sur un principe d'organisation de classe qui maintient une séparation nette entre les individus pouvant s'identifier à la minorité cooptée dans l'enceinte restreinte d'un club et les institutions formelles des démocraties représentatives. C'est à l'abri d'une telle ligne de démarcation que la notion de mythe social donne sens à l'action collective, en saisissant le mode d'expression concrète d'une prise de conscience, d'un engagement, d'une action visant les rapports de pouvoir. Sorel était toutefois conscient des limites du potentiel de transformation sociale incarné par de telles prises de position. À une conception du changement social critiquée pour son spontanéisme trop proche des idéaux romantiques, il adjoignait une analyse politique focalisée sur les institutions formelles qui régissent la vie en société. Les problèmes d'image et de contestation qui marquent depuis peu le Forum de Davos et qui, à terme, hypothèquent son avenir constituent une illustration exemplaire de cette question.

${ }_{12}$ Afin d'éviter tout malentendu, il convient de noter que la filiation entre Sorel et le fascisme tient à une légende tenace dont l'absence de fondement a fait l'objet de nombreux travaux détaillés depuis deux décennies. Voir en particulier: Shlomo Sand, Lillusion du politique. Georges Sorel et le debat intellectuel 19oo, Paris: La Découverte, 1985 et «Sorel, les Juifs et l'antisémitisme», Cahiers Georges Sorel, Vol. 2, 1984, pp. 7-36; Michel Charzat, «Georges Sorel et le fascisme. Eléments d'explication d'une légende tenace», Cahiers Georges Sorel, Vol. 1, 1983, pp. 37-51.
L'analyse procède de la façon suivante. La première section passe en revue la littérature sur le pouvoir informel des clubs délites transnationales en portant une attention particulière aux apports et limites des travaux émanant du champ d'étude de l'économie politique internationale. La seconde section analyse comment la théorie sorélienne du mythe social permet de clarifier le processus de socialisation dans lequel situer le pouvoir informel des clubs. La troisième section est consacrée au cas du Forum économique mondial. Elle s'attache à montrer que l'atmosphère particulière de club exclusif, traduite dans l'expression «esprit de Davos», apparait aujourd'hui comme un mythe social fortement menacé. Après une brève présentation de 
ce qui en a fait son succès, l'analyse porte sur les principales difficultés à transformer le Forum en une organisation se situant à un niveau supérieur d'institutionnalisation. Les conclusions résument l'argument et tirent trois enseignements de l'analyse sorélienne pour appréhender les agents de la mondialisation, leur pouvoir et ses limites.

\section{Le pouvoir informel des clubs d'élites transnationales}

Il existe principalement deux types d'études consacrées aux clubs d'élites transnationales: celles des groupes militants qui les décrient et celles publiées dans le cadre académique des relations internationales. Alors que les premières sous-estiment généralement les enjeux théoriques du pouvoir qu'elles dénoncent et érigent leur critique sur un nombre restreint de sources secondaires, l'apport théorique des secondes sur l'influence des acteurs non étatiques ne prend guère en compte l'environnement concret de leur pratique et, comme pour les premières, n'est que rarement étayée par des recherches empiriques de première main.

Dans un contexte marqué par l'importance de la communication dans les procédures de légitimation, la dissémination d'informations critiques constitue aujourd'hui un des piliers de la stratégie de contestation des groupes militants à l'échelle internationale. Cette stratégie, particulièrement forte dans le monde anglo-saxon, repose sur la fiabilité des informations incriminant l'acteur ciblé par la campagne de communication. Les études de ce type se prémunissent contre les actions en justice en fournissant les preuves de leurs allégations dans un appareil critique étendu ${ }^{13}$. Elles se heurtent toutefois à deux difficultés. D'un point de vue méthodologique, l'accès aux sources n'est pas direct. Généralement par manque de moyens, il dépend des outils de communication produits par l'organisation ciblée (plaquettes de présentation, site internet, etc.), d'articles de journaux et de rapports d'organisations diverses. Ces sources secondaires reproduisent souvent en boucle un nombre très restreint de sources originales, elles-mêmes parfois contestables ${ }^{14}$. D'un point de vue analytique, ces travaux ont tendance à surévaluer le pouvoir réel des organismes placés sous les feux de leur investigation. Les motifs de mobilisation politique à l'origine de ces écrits constituent une part d'explication. La sous-théori-
${ }^{13}$ Belén Balanyá et al., Europe Inc., Marseilles: Agone, 200o; Public Citizen's Global Trade Watch, Davos World Economic Forum: Pricey Corporate Trade Association Loses Its Camouflage, Washington D.C.: Public Citizen, 2002.

14 Dans le cas du WEF, les seules investigations sérieuses sur la controverse relative au chevauchement des activités lucratives et non lucratives de la Fondation présidée par Klaus Schwab sont: «Davos Chief Dabbles in For-Profit Firms, Raising Questions About Forum's Priorities", Wall Street Journal, 27 janvier 200o; «Entrance Fees to the Marketplace of Ideas; High paying Companies Get Some Prime Exposure at High-Powered Davos Conference", Washington Post, 24 janvier 200o. Les informations divulguées par le WSJ sont largement reprises par les ONG et les organes de presse, quelle que soit leur orientation idéologique. À titre d'exemple, l'article du WSJ est cité à 22 reprises et celui du Washington Post à 7 reprises dans la plaquette de dénonciation publiée en 2002 par le Global Trade Watch de l'ONG américain Public Citizen (Public Citizen's Global Trade Watch, op . cit.). 
sation des mécanismes de pouvoir dans des analyses à caractère avant tout pratique en est une autre. Sans forcément reposer sur une véritable théorie du complot, l'impression de conspiration demeure néanmoins.

Dans le cadre académique de l'analyse politique, cela fait plusieurs années que l'étude des clubs d'élites transnationales se rattache à différentes traditions. Pour les travaux qui s'appuient sur la théorie du choix rationnel et qui ont pour questionnement central les processus de décision, il s'agit d'expliquer comment un système de coopération exclusif permet de résoudre des problèmes de coordination et d'efficacité face à la propension des acteurs à maximiser au moindre coût leur pouvoir et leur intérêt ${ }^{15}$. Dans cette perspective, les clubs d'élites transnationales opèrent en parallèle avec les "régimes privés», conçus comme "une congruence sur l'ensemble d'un secteur économique de principes et de normes institutionnalisés dans le cadre d'associations professionnelles, de contrats intersectoriels et de traités ${ }^{16}$. Ils peuvent constituer l'expression organisationnelle d'un régime privé, lorsque leur domaine de compétences recoupe les frontières d'un secteur particulier. C'est par exemple le cas des consortiums actifs dans le domaine des technologies de la communication et de l'information. Si, en revanche, leur fonction n'est pas aussi clairement identifiable, ils empiètent plus facilement sur le domaine diffus de la gouvernance mondiale. Ce concept demeure aujourd'hui très polysémique, à l'instar de la définition retenue par la Commission indépendante du même nom au milieu des années 9o: «L'ensemble des nombreuses méthodes par lesquelles les individus et les institutions, publics et privés, gèrent leurs affaires communes ${ }^{17}$. Les analyses qui assimilent les clubs d'élites transnationales aux groupes d'intérêts privés de la gouvernance mondiale restent divisées sur la discontinuité qui en résulte vis-à-vis des principes de souveraineté, d'anarchie du système inter-étatique et de concurrence du marché ${ }^{18}$. Les clubs bénéficient-ils des qualités intrinsèques d'un réseau particulièrement dense et de la rapidité des prises de

15 Wolfgang H. Reinicke, Global Public Goods, Washington D.C.: Brookings Institution, 1998; Justin Greenwood, "Organized Business and the European Union", in Justin Greenwood and Henry Jacek (eds), Organized Business and the New Global Order, London:

Macmillan, 2000; Margaret

G. Hermann et al., «Who Leads Matters: The Effects of Powerful Individuals", International Studies Review, Vol. 3, $\mathrm{N}^{\circ}$ 2, 2001, pp. 83131. Les fondements théoriques de la méthodologie microécono- mique appliquée par ces approches trouve ses origines dans: James Buchanan, "An Economic Theory of Clubs", Economica, Vol. 32, 1965, pp. 1-14

${ }^{16}$ Virginia Haufler, «Self Regulation and Business Norms: Political Risk, Political Activism", in A. Claire Cutler et al. (eds), Private Authority and International Affairs, Ithaca: SUNY Press, 1999, p. 202.

17 Commission on Global Governance, Our Global Neighbourhood,
Oxford: Oxford University Press, 1995, p. 2.

\footnotetext{
${ }^{18}$ Mark A. Pollack and Gregory C. Shaffer (eds), Transatlantic Governance in the Global Economy, Lanham (MD) and Oxford: Rowman and Littlefield, 2001; Christopher Knill and Dirk Lehmkuhl, «Private Actors and the State: Internationalization and Changing Patterns of Governance», Governance, Vol. 15, $\mathrm{N}^{\circ}$ 1, 2002, pp. 41-63.
} 
décisions qui en découle? ${ }^{19}$ Sont-ils plus spécifiquement l'expression transgouvernementale des contacts noués entre hauts fonctionnaires et experts gouvernementaux tout au long de nombreuses négociations? ${ }^{20}$ Se déploient-ils sur une base plutôt transnationale qui traverse les souverainetés territoriales grâce aux principes de subsidiarité et de délégation de compétence en faveur de l'expertise privée? ${ }^{21}$ Partiellement en réponse à ces questions, les travaux dans le sillage des approches constructivistes en sciences sociales orientent leur démarche vers l'ensemble des institutions où interagissent les acteurs étatiques et non étatiques de la mondialisation. Les clubs sont ainsi conçus comme instances de dialogue, de persuasion et d'identification contribuant à la construction sociale des préférences qui guident l'action collective ${ }^{22}$. Selon Rosenau, un des pionniers les plus éclectiques des recherches consacrées aux nouvelles formes d'autorité sur la scène mondiale, les clubs ne seraient dès lors qu'une modalité parmi d'autres d'un processus de fragmengration, le mot valise par lequel il décrit «les dynamiques d'intégration et de fragmentation qui interagissent de façon simultanée pour donner lieu à de nouvelles sphères d'autorité»" ${ }^{23}$.

Ces différents courants de pensée fournissent, chacun à leur manière, une explication du rôle des élites dans la résolution des problèmes d'action collective et dans la multiplication des sphères d'autorité à l'échelle internationale. Aucun d'entre eux n'aborde toutefois de façon centrale la dimension plus structurelle du pouvoir qu'entretient ce type d'acteurs avec l'État. Il ne s'agit ici ni de gains et de pertes de pouvoir relationnel dans un jeu à somme nulle vis-à-vis de l'État, ni d'instances additionnelles de "gouvernance» dont les fonctionnalités s'ajouteraient «à côté» du rôle traditionnel de l'État sur la scène internationale.

Certains travaux émanant du champ d'étude de l'économie politique internationale ont spécifiquement théorisé l'idée selon laquelle les acteurs étatiques et non étatiques

19 Justin Greenwood, art. cit., pp. 93 ss.

${ }^{20}$ Robert O. Keohane and Joseph Nye, «The Club Model of Multilateral Cooperation and the Problems of Democratic Legitimacy", in Roger B. Porter et al. (eds), Efficiency, Equity, and Legitimacy: The Multilateral Trading System at the Millenium, Washington D.C.: Broo kings Institution Press, 2001; Anne-Marie Slaughter, "The Real New World Order", Foreign Affairs, Vol. 76, 1997, pp. 183-197.

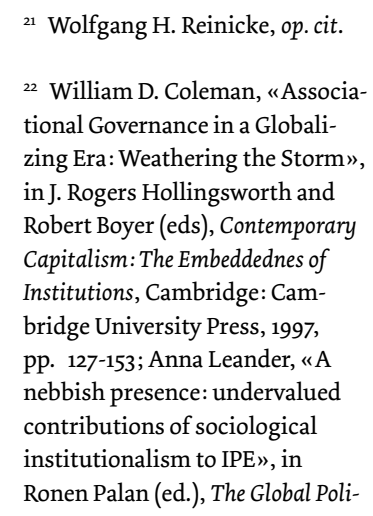
tional Governance in a Globalizing Era: Weathering the Storm", in J. Rogers Hollingsworth and Robert Boyer (eds), Contemporary Capitalism: The Embeddednes of Institutions, Cambridge: Cambridge University Press, 1997, pp. 127-153; Anna Leander, «A nebbish presence: undervalued contributions of sociological institutionalism to IPE", in Ronen Palan (ed.), The Global Poli-

tical Economy: Contemporary Debates, London and New York: Routledge, 2000, pp. 184-196; Thomas Risse, «Transnational actors, networks and global governance», in W. Carlnaes et al. (eds), Handbook of International Relations, London: SAGE, 2001.

${ }^{23}$ James Rosenau, Along the Domestic-Foreign Frontier, Cambridge: Cambridge University Press, 1997, p. 38. 
sont l'expression conjointe d'une même configuration de pouvoir. Comme le relèvent Higgot et ses coauteurs, les «relations entre les acteurs étatiques et non étatiques [sont] parfois conflictuelles, mais souvent aussi symbiotiques» ${ }^{24}$. Dans cette optique, les clubs d'élites transnationales peuvent être appréhendés comme des organisations de classe qui font partie au même titre que l'État du complexe institutionnel qui structure les sociétés capitalistes ${ }^{25}$. Comme le note Sklair, une telle approche met en lumière les agents politiques de la mondialisation: «La mondialisation - tout comme son principal moteur, le capitalisme à l'échelle mondiale - ne surgit pas de nulle part. Elle est conçue, organisée, gérée, promue et défendue contre ses opposants par des groupes de personnes identifiables et qui travaillent dans le cadre d'organisations elles aussi identifiables ${ }^{26}$. En tant qu'organisations de classe, les clubs d'élites transnationales sont parties prenantes aux clivages qui marquent le processus de mondialisation du capitalisme. En contribuant à reproduire l'inégalité structurelle entre ceux qui possèdent les actifs physiques et monétaires des moyens de production et ceux qui par leur travail accroissent les quantités et la valeur marchande de ces actifs, les clubs d'élites transnationales exercent leur pouvoir informel de deux manières conjointes. D’une part, ils contribuent à aiguiser le niveau de conscience des dirigeants économiques et politiques envers les conditions d'existence générales de l'économie capitaliste. D’autre part, ils constituent un lieu privilégié à partir duquel diffuser le plus largement cette vision du monde comme une expression parmi d'autres de l'intérêt général.

${ }^{24}$ Richard Higgott et al. (eds), op. cit., p. 6.

${ }^{25}$ Selon une analyse de classes, les inégalités sociales et politiques ont nécessairement mais pas uniquement - comme point de convergence la matérialité des rapports de production. Cf. Robert W. Cox (with Michael Schechter), The Political Economy of a Plural World: Critical Reflections on Power, Morals and Civilization, London and New York: Rout ledge, 2002, p. 30.

${ }^{26}$ Leslie Sklair, op. cit., p. x.

${ }^{27}$ Stephen Gill, «Structural change and global political economy: Globalizing élites and the emerging world order", in Yoshikazu Sakamoto (ed.), Global Transformation, Tokyo: United Nations University Press, 1994, pp. 179 et 182 .
Selon cette grille de lecture, il ne fait guère de doute que les clubs d'élites transnationales constituent un maillon essentiel d'un pouvoir hégémonique dont l'ambition s'affiche à l'échelle mondiale. Entendu dans son sens gramscien, le concept d'hégémonie exprime la capacité d'une configuration de forces particulière à donner cohérence au pouvoir qu'elle exerce et à le faire accepter sur une base consensuelle le plus largement possible. Gill, une des figures centrales de la théorisation et des recherches dans ce domaine, associe ainsi les clubs d'élites transnationales à un "élément stratégique dans le processus de mondialisation du capitalisme» ou, pour le dire autrement, à l'interface spécifique dans laquelle produire une synthèse politique entre les dimensions territoriales et globales de l'ordre mondial ${ }^{27}$. Comme le souligne van der Pijl, dont les contributions ont aussi fortement marqué ce champ d'étude, ce point de vue ne doit pas être confondu avec la 
thèse du complot: «Les groupes de planification transnationaux ne sont pas un gouvernement mondial conspirateur, mais des organisations de classe réajustant constamment leur position par rapport aux rapports de force auxquels ils se confrontent ${ }^{28}$. En situant les clubs d'élites transnationales dans le cadre plus large des réseaux de fraternisation, d'éducation et de conscientisation mutuelle, Gill et van der Pijl s'appuient non seulement sur les longs développements que Gramsci a consacrés au rôle des intellectuels, mais aussi sur quelques-unes de ses réflexions les plus originales sur l'influence des élites dans le système des démocraties représentatives. Pour Gramsci, le principe libéral du suffrage universel n'est en effet pas en mesure de répondre au fait que «les idées et les opinions ne naissent pas spontanément dans le cerveau de chaque individu: elles ont un centre de formation, d'irradiation, de diffusion, de persuasion ${ }^{29}$. C'est dans cette optique que les clubs d'élites transnationales apparaissent comme un objet privilégié pour étudier le rôle crucial d'une classe spécifiquement transnationale dans l'histoire longue du capitalisme. Pour van der Pijl, le WEF succéderait ainsi à d'autres organisations qui l'ont précédé, comme la franc-maçonnerie, le réseau gravitant autour de la Round Table à l'apogée de l'Empire britannique ou les conférences de Bildeberg tout au long de la guerre froide et au-delà. Mais selon lui, à la fin des années 9o, le WEF constituait bel et bien «le groupe de planification transnational le plus accompli, [...] une véritable Internationale du capital $»^{30}$.

Il est aujourd'hui reconnu que les approches plus ou moins dérivées des écrits de Gramsci soulignent de façon convaincante le besoin d'intégrer les dimensions matérielles et normatives, coercitives et consensuelles dans l'analyse des enjeux structurels du pouvoir à l'échelle mondiale ${ }^{31}$. Ces approches offrent en outre des pistes de réflexion fécondes sur le rôle éducatif de l'État et les nombreuses institutions non étatiques dans ce processus de socialisation. Si elles fournissent les outils pour théoriser la cohérence dans laquelle situer la relation entre acteurs étatiques et non étatiques, seuls un nombre restreint de travaux s'en sont inspirés pour mener des études empiriques approfondies. À part celles déjà citées de Gill sur la Commission trilatérale et de van Apeldoorn sur la European Rountable of Industrialists, les références sont fréquentes, mais toujours brèves, sans investigation préalable substantielle et, comme les pamphlets des groupes militants, tendent à reproduire en boucle un nombre ${ }^{28}$ Kees van der Pijl, Transnational Classes, op. cit., p. 134.

29 Antonio Gramsci, Cahiers de prison: Cahiers 10, 11, 12 et 13, Paris: Gallimard, 1978, p. 421 (Cahier 13 $\S 3)$.

30 Kees van der Pijl, op. cit., p. 133.

${ }^{31}$ Marie-Claude Smouts (dir.), Les nouvelles relations internationales, Paris: Presses de Sciences Po, 1998, pp. 20-21; Pierre de Senarclens, La politique internationale, Paris: A. Colin, 2002, pp. 197-198; Jean-Christophe Graz, «Les nouvelles tendances de l'économie politique internationale", Annuaire français de relations internationales, Vol. 1, 2000, pp. 557569; Christian Chavagneux, L'économie politique internationale, restreint de sources originales. Le nombre limité d'institu- Paris: La Découverte, à paraître. 
tions dont l'incidence dépasse le rôle traditionnel de lobby constitue une part d'explication. La difficulté d'accès à l'information également. Enfin, pour van der Pijl, c'est une stratégie de recherche délibérée qui évite de survaloriser une instance de pouvoir par rapport à une autre et garde une vue d'ensemble sur la continuité historique, les multiples chevauchements et les métamorphoses continuelles des organisations de planification stratégiques du capitalisme sur le long terme ${ }^{32}$. Il reste qu'un organisme comme le WEF est suffisamment central pour mériter à lui seul une analyse détaillée. Plus fondamentalement, même dans le cas des rares études citées, il manque une théorisation du type de pratique spécifique qui a cours dans le cadre même des clubs. Le renvoi à la fonction gramscienne de l'hégémonie et l'évidence des réseaux d'élites à

76 l'échelle internationale ne suffisent en effet pas à expliquer pourquoi certaines platesformes et certains réseaux sont plus courus que d'autres. La question demeure donc: comment identifier ce qui rend concrètement possible la fonction hégémonique de certaines instances et non pas d'autres? Pour y répondre, il convient de revenir sur le processus de socialisation dans lequel s'exerce le pouvoir informel des clubs.

\section{Mythe social et pouvoir hégémonique}

Le mythe créé par le rassemblement d'un ensemble de personnes appartenant toutes plus ou moins intimement aux sphères dirigeantes constitue aussi bien la force que

${ }^{32}$ Kees van der Pijl, op. cit., p. 134.

33 Cette perspective nécessiterait un travail sémiologique inspiré notamment par les écrits de Barthes sur le mythe comme "parole dépolitisée»; voir: Roland Barthes, Mythologies, Paris: Seuil, 1957, pp. 229 ss.

34 Georges Sorel, Réflexions sur la violence, Paris: Seuil, 1990; Antonio Gramsci, op. cit., pp. 289 ss et 354 ss.

35 Enrico Augelli and Craig Murphy, «Consciousness, myth and collective action: Gramsci, Sorel, and the ethical state", in Stephen Gill and James H. Mittelman (eds), Innovation and Transformation in International Studies, Cambridge: Cambridge University Press, 1996, p. 29

${ }^{36}$ Georges Sorel, Réflexions sur la violence, Paris: Seuil, 199o, pp. 29 , 8 et 216 . la faiblesse des clubs d'élites transnationales. Le recours au concept de mythe ne vise pas ici à restituer la part d'illusion - souvent massive - que mobilisent ces événements ${ }^{33}$. Il renvoie au concept de mythe social forgé par Georges Sorel dans ses Réflexions sur la Violence (1906) et repris par Gramsci dans certains de ses écrits sur l'hégémonie ${ }^{34}$. Comme l'indiquent Augelli et Murphy, pour le courant qui s'inspire de la pensée gramscienne dans les relations internationales, le concept de mythe social se révèle particulièrement fécond pour appréhender la façon dont les dirigeants tentent de «motiver une action politique collective susceptible de préserver ou au contraire de transformer un aspect majeur de l'ordre mondial» ${ }^{35}$. L'expression de mythe social revient à plusieurs reprises sous la plume de Sorel, même s'il n'en offre pas de définition formelle. Selon les passages, elle décrit «l'expression des convictions profondes d'un groupe dans le langage du mouvement», "l'expression de la volonté d'agir», ou simplement une idéologie «riche en sublime» ${ }^{36}$. Comme le 
relèvent divers exégètes, la matrice de la théorie sorélienne du mythe se trouve chez Vico. Dans la Science nouvelle, publiée en 1744, le philosophe napolitain appréhende le mythe comme une propension de la pensée primitive à transfigurer un fragment d'histoire humaine en image mobilisatrice représentative de l'avenir ${ }^{37}$. La force d'un mythe social repose sur l'intensité de la prise de conscience suscitée par une passion collective. Une telle expérience démarque clairement ceux qui en font partie et se projettent dans l'avenir de ceux qui n'en font pas et s'obstinent à préserver le passé. Ce principe de scission agit comme révélateur des intérêts de classe.

Le concept de mythe social offre les moyens de saisir le mode d'expression concrète d'une conscience collective dans un contexte de mobilisation sociale très condensée dans le temps, le lieu et l'action. Selon Sorel, alors que les premiers chrétiens tenaient le mythe de jugement dernier comme moteur de leur lutte contre l'Empire romain, au tournant du $\mathrm{XX}^{\mathrm{e}}$ siècle, la grève générale reconduit le même procédé en galvanisant la conscience de classe des travailleurs et en renforçant leur ressort révolutionnaire. De ce point de vue, la force de la grève générale se trouve dans le refus de poursuivre une longue histoire de compromis boiteux et dans la mise à nu des intérêts de classe: le mythe social "s'enferme tout entier» pour ne plus faire qu' "une indivisible unité» - et Sorel d'ajouter, «la société est bien divisée en deux camps, et seulement en deux, sur le champ de bataille» ${ }^{3}$.

Par effet de miroir, la pratique des clubs d'élites transnationales repose sur le même principe. En rendant manifeste la ligne qui sépare les inclus des exclus, les clubs d'élites reconduisent le clivage entre ceux qui s'estiment suffisamment importants pour interagir derrière des portes closes et ceux qui n'ont pas leur place dans ce genre d'arène exclusive. La mobilisation des "forces créatrices" prend place dans un espace confiné et coupé de la sphère publique. Composé d'acteurs étant de facto en position de pouvoir, le milieu privé des clubs d'élites est investi d'une capacité à apporter des changements dans la société qui les entoure. L'accès exclusif et non discriminatoire à un réseau particulièrement

37 Jules Monnerot, «Georges Sorel ou l'introduction aux mythes modernes", in Science et dense entretient l'idée d'être associé de près ou de loin à l'avenir du monde. C'est ce qu'évoque l'image maintes fois relayée de «lieu de rencontre des maîtres du monde».

Si le concept de mythe social exprime bien la dimension concrète du pouvoir mobilisateur des clubs d'élites transnationales, il renferme aussi les éléments susceptibles d'en expliquer les limites. Il relaie en effet une conscience de la société: mélanges en l'honneur de Raymond Aron, Vol. 1, Paris: Calmann-Lévy, 1971 [cité in Jacques Juillard, «Préface: Au risque de penser", in Georges Sorel, Réflexions sur la violence, op. cit., pp. vi-vii]; Robert W. Cox, op. cit., pp. 51-52.

${ }^{38}$ Georges Sorel, op. cit., pp. 120, 126. 
conception spontanée du changement social et mise sur une pratique coupée des institutions formelles de la société. Dans cette optique, il circonscrit le politique au domaine du possible partagé par les seuls individus inclus dans l'action. Il peut certes être l'expression de leur "volonté d'agir», mais pas celle des forces sociales qui les ont placés là où ils sont. De même, il peut déboucher sur des initiatives, létablissement de groupes de travail et des «task forces». Mais pour dépasser les seules retombées bureaucratiques et symboliques, il doit se confronter au processus formel du droit, des institutions politiques et de l'État. Dans son essai sur l'Affaire Dreyfus - publié quelques années après les Réflexions - Sorel reconnaissait qu'une conception trop spontanéiste du mythe social était vouée à l'échec. Il insistait au contraire sur la relation qui prévaut entre la prise de conscience provoquée par un mythe social et la planification à long terme requise pour entraîner une transformation en profondeur des institutions qui régissent la société. De son point de vue, le droit public demeurait la cible à ne pas perdre de vue: «Lorsque le calme est complet et que l'avenir se présente comme assuré, on s'occupe d'élever la nouvelle génération dans l'idée que le nouveau régime est fondé sur des théories de droit public incontestables»39.

Il apparait clairement que la lecture que fait Sorel lui-même du mythe social nous conduit à donner sens à ce concept dans sa relation avec la problématique gramscienne de l'hégémonie. Quoique contestée par Gramsci, cette interprétation est corroborée par le fait que les deux auteurs cherchent à lier dans leur analyse les deux moments de la prise de conscience d'une part, et de l'action d'autre part, du consensus et de la coercition ou, pour reprendre une expression gramscienne consacrée, de la guerre de position et de la guerre de mouvement ${ }^{40}$. Les écrits de Sorel ont durablement influencé le cadre de pensée du jeune Gramsci. Plus tard, en dépit de la bolchevisation du Parti communiste italien dans les années 20, mettant à l'index toute forme de spontanéisme prolétarien, Gramsci continue à s'y référer pour développer ses concepts, en particulier celui de «bloc historique» dont la place est centrale dans sa théorie de l'hégémonie ${ }^{41}$. Entendu comme congruence de forces sociales partageant un niveau de conscience suffisamment élevé pour s'organiser politiquement, un bloc historique est

39 Georges Sorel, La révolution dreyfusienne, Paris: Rivière, 1911 ( 2 éd.), p. 20

40 Antonio Gramsci, op. cit., pp. 354 ss.

${ }^{41}$ Michel Charzat, "À la source $\mathrm{du}$ ‘marxisme` de Gramsci», Cahiers de l'Herne. Georges Sorel, 1986, pp. 213-222. le passage obligé pour exercer un pouvoir hégémonique fondé sur le consensus plus que la coercition.

La relation mutuelle entre l'engagement individuel associé au mythe social et le travail politique d'une direction hégémonique s'inscrit dans un continuum. Les bornes qui le délimitent sont, d'un côté, la vanité pure d'acteurs sociaux qui se prennent pour les agents autonomes 
du changement et, de l'autre, les institutions publiques autour desquelles se noue de façon consensuelle la capacité d'une configuration de forces particulière de contrôler les bases matérielles et financières de la vie en société. Les potentialités mobilisatrices des mythes sociaux peuvent ainsi apparaître comme condition nécessaire de la conquête de l'hégémonie, mais non suffisante. Comme le note Charzat à l'issue de son analyse des liens de filiation entre Gramsci et Sorel, «de grandes passions collectives [...] peuvent accélérer l'éclosion de la nouvelle société, à condition d'être précédées d'un travail patient et systématique d'investissement des institutions existantes, par l'éclosion d'institutions autonomes ${ }^{42}$.

En quoi les développements qui précèdent contribuent-ils à une démarche focalisée sur les limites du pouvoir des clubs d'élites transnationales? En premier lieu, la capacité de groupes informels et élitaires à exercer un pouvoir réel de planification stratégique au-delà des consultations discrètes, des arrangements privés et des effets d'annonce sera toujours mise à l'épreuve des institutions politiques et de la mobilisation sociale. Quelle que soit l'intensité de la conscience de classe des acteurs impliqués, la volonté collective censée être activée dans le confinement des clubs doit, à un moment ou un autre, se confronter aux forces sociales qui lui sont extérieures. Dans l'environnement institutionnel des sociétés démocratiques modernes, la transposition d'un projet défendu à l'abri de portes closes est ainsi le résultat de processus complexes ayant souvent des conséquences imprévues. En second lieu, dans le cadre de réflexion gramscien, le sens à attribuer aux limites de l'influence des clubs élitaires est à chercher dans les formes non hégémoniques de pouvoir. Pour l'auteur des Cahiers de prison, cela renvoie au transformisme, ce genre particulier de "révolution passive» où les transformations sociales excluent une mobilisation active des forces populaires ${ }^{43}$. Le transformisme repose sur une coalition d'intérêts qui cristallise les problèmes plus qu'elle ne les résout en cooptant les dirigeants des groupes d'opposition et en assimilant les éléments les plus subversifs de leur discours. C'est à partir de ce type de conception managériale du pouvoir que les groupes de planification élitaires élaborent aujourd'hui toutes sortes de réformes visant à rééquilibrer les inégalités qui émanent du processus de mondialisation du capitalisme ${ }^{44}$. Certaines initiatives peuvent mener à des résultats tangibles après avoir rejoint les canaux plus officiels de la politique. Mais ce système détourné d'influence peut rarement faire l'économie de procédures plus intégrées et socialisées du pouvoir pour canaliser les relations entre l'économique et le politique sur le plan mondial.

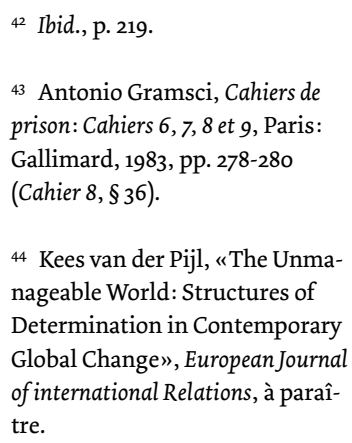
Global Change», European Journal of international Relations, à paraître. 
C'est dans ce cadre qu'il convient de placer le pouvoir informel d'une organisation comme le Forum économique mondial. Dans les lignes qui suivent, il s'agira d'évaluer quel mythe social a servi de soubassement à l'histoire du WEF, quelles sont ses relations avec les diverses institutions politiques du monde contemporain et, enfin, en quoi les problèmes que rencontre le Forum depuis quelques années reflètent les limites intrinsèques d'un pouvoir coupé des institutions représentatives de la vie publique.

\section{Le cas du Forum économique mondial}

L'histoire officielle du Forum économique mondial évoque fièrement la façon dont le mythe social des réunions annuelles de Davos peut déboucher sur des transformations d'envergure à l'échelle mondiale ${ }^{45}$. La recette du succès tient dans les trois unités du théâtre classique. Ce sont elles qui ont la capacité de transporter dans un état fusionnel les hautes sphères économiques et politiques de ce monde. L'unité de lieu se joue dans l'espace clos d'une station alpine difficile d'accès. Par métonymie, Davos est le Forum économique mondial pour une semaine chaque année, à fin janvier. L'unité de temps, en raison de l'accès difficile à l'espace clos, se déroule sur une durée incompressible et relativement longue par rapport à l'agenda quotidien des participants. À part les super-VIP qui viennent et repartent en hélicoptère, les participants restent en général pour trois jours au moins. Enfin, l'unité d'action tient dans l'absence des distractions propres aux grandes capitales qu'ils fréquentent habituellement. À Davos, à part skier, il n'y a rien d'autre à faire durant cette période.

L'expression consacrée pour saisir cette atmosphère particulière de club propice à la magie des rencontres impromptues est «l'esprit de Davos» ${ }^{6}$. La passion collective suscitée par la densité inégalable du réseau de contacts à disposition est, dans l'imagerie du Forum, liée à la capacité d'accomplir sa mission: «résolu à améliorer l'état du monde». Les pratiques mises en œuvre dans le cadre du Forum sont toutefois loin de correspondre à celles d'une organisation vouée à la seule planification stratégique du capitalisme mondial. À maints égards, le Forum s'apparente plus modestement à un espace polymorphe d'intermédiations tourné vers les nouvelles frontières du capitalisme. La raison d'être de participer aux activités du

${ }^{45}$ Voir http://www.weforum.org/ site/homepublic.nsf/Content/Our + History

${ }^{46}$ Voir la présentation de l'édition 2003 du Forum: http://www. weforum.com/site/homepublic. nsf/Content/Annual +Meeting $+2003 \% 5$ CAbout+the+Annual + Meeting
Forum s'étend tout au long du spectre qui relie le mythe social mondain à la direction hégémonique du monde. Entre pure vanité et planification stratégique, l'homme de Davos peut être guidé par la perception de l'air du temps, des sujets à la mode, l'établissement de contacts clés, le déblocage de négociations en cours ou linfluence sur l'orientation de telle ou telle réglementation ou légis- 
lation ${ }^{47}$. Dans l'essai cynique sur son expérience davosienne, le rédacteur en chef de la revue américaine Harper's Magazine associe même le rituel de Davos à l'Agonie de Mammon dans le Parsifal de Wagner ou aux saynètes médiévales dans lesquelles des personnages en costume jouent les mystères de Pâques d'Oberammergau ${ }^{48}$. Mais pour un habitué comme Jacques Attali, l'ancien conseiller spécial du président français François Mitterrand, le Forum est avant tout «un hôtelier qui permet de gagner du temps; c'est un économiseur de voyages d'affaires» ${ }^{49}$. Selon un universitaire qui compte lui aussi parmi les habitués, le spectre des motivations des participants est certes plus large, sans pour autant embrasser le sens de l'engagement invoqué par les organisateurs: «Personne ne vient ici pour réfléchir; il y a ceux qui sont là pour faire du business et qu'on ne voit pas; il y a ceux qui, au contraire, se montrent partout pour multiplier les effets d'annonce; et tout le monde participe à un effet de miroir dans sa tentative de mesurer l'air du temps ${ }^{50}$. En définitive, c'est souvent l'ensemble de ces raisons, avec des pondérations variables, qu'invoquent les participants lorsqu'on les questionne sur ce point. Au-delà des limites intrinsèques du pouvoir que peut incarner un tel club d'élites transnationales, les responsables du Forum sont bien conscients que ce qui a fait le succès de la recette est en train d'en faire son échec. Aujourd'hui, c'est le mythe social de la réunion annuelle de Davos qui est lui-même menacé.

Sur le plan interne, le gigantisme pris par la manifestation limite les opportunités offertes par le réseau de Davos. Au cœur du mythe de «l'esprit de Davos» se trouve le principe que tous les porteurs du «badge blanc» font partie de la même communauté et peuvent entrer en contact les uns avec les autres sans autre intermédiaire que le hasard des rencontres et les bornes informatiques prévues à cet usage dans la zone réservée aux seuls participants du Forum. Un universitaire invité pour ses compétences particulières sur l'Asie centrale ou sur la physique des particules peut ainsi espérer entrer en contact (et plus si entente) avec le fondateur de Microsoft ou le directeur de Nestlé. Inversement, Michael Dell, le fondateur et directeur de l'une des plus grandes entreprises informatiques

47 L'expression «homme de Davos" peut paraître sexiste, mais elle renvoie au fait que jusqu'en 2003, les femmes représentaient moins de $10 \%$ des participants; voir World Economic Forum, Women Leaders Initiatives, en ligne: http://www.weforum.org/ site/homepublic.nsf/Content/ Women+Leaders+Initiative

du monde, peut s'autoriser à une discussion à bâtons rom- ${ }^{48}$ Lewis Lapham, op. cit., p. 58. pus avec la directrice d'une organisation de commerce équitable en lui confiant qu'il a ainsi l'impression de pouvoir «prendre l'air du temps et de sortir de sa bulle pour aller vers le monde réel » ${ }^{51}$. En raison de la taille de l'événement et du nombre de rendez-vous organisés à l'avance, les possibilités de bénéficier de ce principe d'opportunité

\footnotetext{
49 Entretien avec Jacques Attali, Paris, 26 avril 2001.

5o Entretien confidentiel, Davos, 25 janvier 2001.

${ }^{51}$ Entretien confidentiel, 12 avril 2001.
} 
dépendent en fait largement de l'insertion préalable dans le réseau. Comme l'explique par euphémisme l'un des participants, «l'usage veut qu'on ne refuse pas un contact, sauf si l'on est ‘full booked`; ça dépend donc des rendez-vous pris à l'avance» ${ }^{52}$. Or, à en croire un vieux routinier comme Jacques Attali, le meilleur moyen d'organiser son temps est «d'accepter à l'avance plus d'invitations à déjeuner et à dîner que le nombre de repas existant et choisir au dernier moment la meilleure opportunité» ${ }^{53}$. Les témoignages concordent sur le fait que Davos abolit beaucoup moins les hiérarchies qu'on ne le prétend souvent.

La stratégie de croissance de la fondation dans les années 90 a également accru la visibilité de la face cachée du mythe. Les réunions privées des dirigeants industriels, financiers et politiques du monde qui se tiennent en marge des rendez-vous d'affaires et des panels de discussion ne sont pas nouvelles, de loin pas. Mais le poids des intérêts représentés dans le cadre de ces séances et la débauche de moyens engagés pour faire des affaires ne semblent proportionnels qu'à l'insistance avec laquelle les organisateurs du Forum véhiculent l'image de réunions désintéressées. Ce problème, lancinant pendant des années, est devenu crucial à partir du milieu des années 90 - et ce en tout cas jusqu'à la relative cure d'abstinence imposée à l'édition 2003. Même les quotidiens les plus réputés dans le monde des affaires en font leurs choux gras. En 2000, le Wall Street Journal et le Washington Post lèvent le voile sur les relations entre la fondation à but non lucratif qui chapeaute les activités du Forum, les activités très lucratives qu'elle autorise et les intérêts privés qu'en retire son président, Klaus Schwab ${ }^{54}$. Depuis plusieurs années, le Financial Times ironise sur le manque de crédibilité dans la prétention du WEF à incarner l'intérêt général. Ainsi, lors de l'édition 2002, il identifiait ce problème comme le «facteur Bono", en référence au chanteur du groupe de rock irlandais U2 venu à New York exhorter les grands de ce monde à se préoccuper plus sérieusement du sort de l'Afrique et de l'épidémie de sida ${ }^{55}$. Le facteur Bono est alors défini comme «l'inquiétude parmi les quelque 2000 délégués de Davos à pouvoir démontrer qu'en dépit de leur extraordinaire richesse et puissance collective [...], ils se préoccupent de l'influence néfaste de la pauvreté dans le monde et veulent faire quelque chose à ce propos. C'est la mauvaise conscience de Davos. [...] Et pourtant, il reste difficile de s'extraire du sentiment que, malgré le sérieux des discussions et le nombre de sourcils froncés, ce qu'est vraiment Davos pour la plupart des participants demeure l'occasion de nouer des contacts et faire des affaires» ${ }^{56}$.

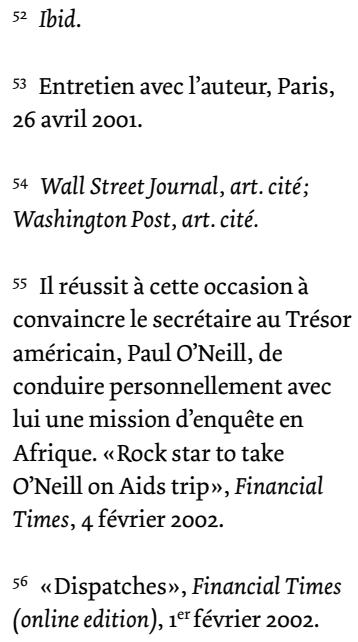

56 "Dispatches", Financial Times (online edition), $1^{\text {er }}$ février 2002. 
La taille de l'événement et la détérioration de l’image publique du Forum entraînent avec elles un risque de désaffection de participants contrariés par un gigantisme et un esprit de forteresse trop contraires à «l'esprit de Davos». Déjà en 2001, de nombreux participants à la réunion annuelle faisaient part de leur réserve à l'égard de l'ampleur prise par les mesures de sécurité et se demandaient s'ils continueraient d'y participer à l'avenir57. En 2003, les organisateurs ont choisi de placer la rencontre annuelle sous le thème de la confiance, mais c'est bien le manque de celle-ci dans la gestion interne des entreprises et dans l'image du monde véhiculée par le Forum qui semble avoir induit une participation plus faible et moins prestigieuse, ainsi qu'une ambiance nettement plus morose qu'à l'accoutumée ${ }^{58}$. Cette tendance fragilise la base financière du Forum et hypothèque sérieusement son avenir. Car, comme l'explique sans détour l'ancien comptable responsable de l'organisation des réunions annuelles, "c'est le business qui paie pour tous les autres invités» ${ }^{59}$. Les milieux d'affaires paient en effet les finances d'inscription, les cotisations de membre et les droits associés aux divers contrats de partenariats conclus avec la fondation.

Sur le plan externe, la densité, la centralité et le confinement du réseau voulu par ce genre d'événement constituent une cible privilégiée pour le mouvement d'opposition à la mondialisation néolibérale. Après la mobilisation contre le projet d'Accord multilatéral sur les investissements en 1998, les grandes manifestations contre le G7 à Cologne en 1999 et la débâcle de la réunion ministérielle de l'OMC à Seattle à la fin de la même année, l'édition $2000 \mathrm{du}$ Forum a pour la première fois fait face à une opposition bien organisée. L'ONG suisse tiers-mondiste Déclaration de Berne a mis sur pied un contre-forum sur la base d'un collectif international d'ONG intitulé Public Eye on Davos. En reprenant à leur compte les principes de liberté, de démocratie et de transparence, les organisateurs ont su gagner l'attention des organes de presse. L'image d'un groupe de militants en habits de ski et rivés à leurs jumelles constitua une pièce de choix pour l'illustration des articles de la presse internationale qui, pour une fois, apportaient une touche d'originalité par rapport au rituel annuel du 57 Entretiens divers avec l'auteur. Forum. Parallèlement, des manifestations ont eu lieu dès 1999 et ont rapidement pris une ampleur considérable. Les difficultés d'accès à la station grisonne dictées par sa situation géographique et les mesures de sécurité draconiennes prises par les autorités helvétiques ont empêché le déroulement d'actions d'envergure sur les lieux mêmes du Forum. Mais lors de l'édition 2001, des heurts violents ont opposé les forces de l'ordre aux manifestants refoulés jusqu'à

58 "Investment Bankers Fell Like Scapegoats", Wall Street Journal Europe, 27 janvier 2003, p. A4; "Challenges on every front", The World: 2003. The World Economic Forum annual meeting opens today in Davos; Financial Times Special Report, 23 janvier 2003, p. 1; "(Pooh) bearish", Observer in Davos, Financial Times, 27 janvier 2003.

Zurich, la capitale financière du pays. En 2003, le même scénario s'est répété, cette fois-ci à Berne.

59 Entretien confidentiel, Genève, 14 décembre 2000. 
Face à ces pressions internes et externes, l'avenir du Forum parait incertain. Ses dirigeants ont mis en place deux types de mesures pour faire face à ces difficultés. En premier lieu, pour reconduire le mythe social des réunions annuelles, le maintien de l'atmosphère de club exclusif à l'abri du monde extérieur doit pouvoir être assuré quel qu'en soit le coût. Alors que la facture pour assurer la sécurité de l'événement ne se montait qu'à quelques centaines de milliers de francs suisses en 1997, la montée en puissance de l'opposition au Forum a rapidement fait grimper les dépenses de sécurité. En 2001, elles atteignirent plusieurs millions de francs suisses. Pour l'édition 2002, les conséquences dramatiques des événements du 11 septembre 2001 se sont ajoutées aux craintes de voir grossir encore la montée en puissance des manifestants de l'édition 2001. Après le 11 septembre, les négociations sur le partage de la facture des frais de sécurité n’avaient toujours pas abouti et les organisateurs redoutaient que l'augmentation vertigineuse des primes d'assurance concernant la participation à l'événement dissuadât encore plus les habitués (en particulier les 30\% de ressortissants des États-Unis). Pour la première fois en plus de trente ans, les organes dirigeants du Forum économique mondial décidèrent d'abandonner Davos et d'organiser la réunion annuelle à New York. L'entreprise de relation publique associée au déménagement de l'édition 2002 à New York fut menée de main de maître pour l'associer à une démonstration de solidarité avec une ville blessée par les attentats meurtriers contre les deux tours du World Trade Center. Mais c'est le climat d'incertitude organisationnelle qui motiva avant tout le déménagement. L'expérience fut d'ailleurs mitigée en raison de la difficulté d'exporter dans un tel lieu l'atmosphère de club: dans une ville mondiale comme New York, impossible de reproduire les unités de lieu, de temps et d'action chères à Davos ${ }^{60}$. Les participants ont d'autres affaires à régler et se croisent moins dans les enceintes mêmes du Forum. Quoi d'étonnant, dès lors, si la Confédération suisse, le canton des Grisons et la commune de Davos

6o Guy de Jonquières, Lionel Barber, Hugh Carnegy, "Davos has <lost> its rarefied atmosphere», Financial Times (online edition), 4 février 2002.

61 «Le retour à Davos du World Economic Forum est programmé pour 2003", Le Temps (Genève), 19 janvier 2002; "Mehr Rechte für Polizei», Tages Anzeiger (Zürich), 29 novembre 2001; «Finanzierung der WEF-Sicherheit noch nicht geklärt», Sonntagszeitung (Zürich), 11 novembre 2001; «Swiss Resort Won't Miss Its Famous Guests", Los Angeles Times, 8 novembre 2001, A1, p. 17. se sont empressés de fournir aux organisateurs du Forum des engagements financiers et logistiques pour assurer le retour à Davos en 2003. Environ neuf dixièmes des 14 millions de francs suisses inscrits au budget de la sécurité de l'édition 2003 ont été assurés par les pouvoirs publics. Alors que le Forum semble non exportable (le choix d'une autre station reculée ne ferait que déplacer le lieu d'une cible facilement identifiable pour les mouvements de contestation), le coût social et financier pour assurer sa sécurité continue à faire l'objet d'un vif débat ${ }^{61}$.

En second lieu, les dirigeants de la Fondation s'emploient à transposer les activités du Forum à un niveau 
supérieur d'institutionnalisation. L'objectif est de dépasser le côté éphémère du mythe social de la réunion annuelle de Davos et de créer des prolongements institutionnels que je qualifierai ici d'ateliers hégémoniques. Par cette notion, il faut entendre de petits groupes émanant de l'élite transnationale sollicités à développer entre eux des projets dont l'impact immédiat sur le cours d'un problème particulier vaudrait comme "pratique exemplaire» (best practice) des solutions consensuelles à trouver aux situations conflictuelles. Pour les dirigeants de la Fondation, il s'agit d'appliquer lege artis la doctrine de l'administration des affaires: le Forum, nous dit-on, est en train de se transformer en une institution "plus orientée vers les résultats", passant d'une fonction de "facilitation" à celle de "catalyseur», et mieux réaliser ainsi tout le potentiel d'une "organisation de connaissance et de processus» ${ }^{62}$. Les participants aux réunions sont donc bombardés de documents préparatoires sous forme de notes de synthèse, de chiffres et de textes originaux produits par les grands cabinets de conseil, comme Oxford Analytica ou Accenture. Le secrétariat de la fondation est continuellement réorganisé sur la base de nouvelles structures, telles que le "Centre pour l'agenda global», celui pour les «industries globales», ou encore celui pour les «stratégies régionales» ${ }^{63}$. Des task forces et initiatives de toutes sortes sont lancées à grand renfort de publicité. Même si beaucoup d'entre elles ont vite été perdues de vue, quelques-unes réussissent à rivaliser avec d'autres projets en montant un dossier suffisamment solide pour influencer l'agenda politique. Dans ces eaux mouvantes, le navire amiral autoproclamé du WEF reste le document soumis au Sommet d'Okinawa du G7 en 2000 par le groupe en charge de la «fracture numérique»; il aurait constitué les grandes lignes du programme d'inclusion des pays pauvres dans la nouvelle ère de l'économie numérique adopté à cette occasion. Mais depuis l'éclatement de la bulle internet, on n'en a plus guère entendu parler.

Trois problèmes semblent grever les chances de succès de la réorientation stratégique du Forum. Tout d'abord, il ne faut pas oublier que c'est toujours le réseau de contacts et la conduite des affaires qui constituent la première motivation à payer le ticket d'entrée aux activités du ${ }^{62}$ Entretien avec Charles Forum. Les ateliers hégémoniques peuvent tout au plus compléter, mais aucunement supplanter les réunions annuelles ou régionales. Ensuite, une entreprise hégémonique suppose de rompre la scission de classe sur laquelle tout le succès de Davos a été fondé. L'ambiance calfeutrée de club élitaire devrait alors être en mesure d'élargir le cercle de personnes invitées en y incluant les représentants d'intérêts subordonnés comme les dirigeants syndicaux McLean, directeur de la communication, Forum économique mondial, Genève, 21 décembre 2000; Klaus Schwab, «Opening Address to the Annual Meeting 2001", Davos, 25 janvier 2001.

${ }^{63}$ Un des projets du Forum s'intitule même «Global Agenda Atelier»; voir http://www.weforum. org/site/homepublic.nsf/Content/ Global+Agenda+Atelier 
ou les représentants d'ONG militantes. Dans un contexte d'hostilité croissante, le Forum a invité pour l'édition 2000 une poignée de dirigeants d'ONG à se joindre aux cinq ou six dirigeants syndicaux qui comptaient déjà parmi ses habitués. En 2001, ils étaient une trentaine, soit environ $1 \%$ des participants, et incluaient certains des critiques les plus en vue du moment, à l'enseigne de Thilo Bode de Greenpeace, de Martin Kohr du Third World Network, de Lori Wallach de Public Citizen ou Vandana Shiva et la Research Foundation for Science. Mais en 2002, après avoir condamné publiquement la répression exercée à l'encontre des manifestants, soit ils n’ont pas reçu de nouvelle invitation, soit ils l'ont déclinée ${ }^{64}$. À titre d'exemple, une des raisons qui ont motivé Greenpeace à décliner l'invitation fut sa découverte de ne pas avoir été conviée à plusieurs réunions de la task force à laquelle elle était censée participer aux côtés de l'industrie automobile pour débattre du rôle de cette industrie dans la gestion du changement climatique ${ }^{65}$. Pour ces dirigeants, Porto Alegre s'était déjà substitué à New York ou Davos comme le lieu où il fallait être.

Enfin, les nouveaux processus institutionnels du Forum ne se contentent pas d'exclure des interlocuteurs stratégiques, ils peinent aussi à forger de façon solide une prise de conscience parmi ceux qui y participent. La plupart des initiatives reposent sur la force de travail des «Global leaders of tomorrow» (GLT). Cette catégorie de participants est recrutée parmi des individus de moins de 40 ans seetant particulièrement distingués dans le domaine des affaires, de la politique ou du point de vue académique, culturel ou religieux. La centaine de nouvelles personnes cooptées chaque année a le privilège de participer aux activités du Forum à titre gratuit, mais cela seulement pendant cinq ans. Pour continuer à faire partie du cercle d'initiés au-delà de ce délai, soit la compagnie qu'ils dirigent ou à laquelle ils appartiennent obtient (ou a déjà) le statut de membre et paie ses cotisations, soit ils continuent d'être invités en tant que "forum fellow». Mais s'ils n'ont pas contribué de façon satisfaisante à la valeur ajoutée du Forum, ils sont exclus. C'est leur expertise managériale qui constitue ainsi leur droit d'accès à la strate

${ }^{64}$ Entretiens téléphoniques et correspondances avec l'auteur, janvier 2002.

65 Lettre de Geird Leipold, directeur exécutif de Greenpeace International, à Klaus Schwab, 10 janvier 2002 (publiée sur le site internet de Greenpeace: http:// www.greenpeace.org/politics/we /wefmtg16jan2oo2.html).

${ }^{66}$ Entretien confidentiel, 12 avril 2001. la plus élevée de l'élite transnationale. Or, ces règles du jeu aiguisent les ambitions personnelles. Comme l'explique une participante: «Il y a trop d'ego, avec des personnes qui cherchent à tirer la couverture à elles. Dans ces conditions, il vaut mieux rester prudent en matière de distribution d'information; ces personnes ne ratent pas une occasion de l'utiliser à leur propre compte pour mieux se profiler» ${ }^{66}$. Ce manque exemplaire de conscience de classe empêche les GLT de dépasser leurs intérêts individuels pour prétendre embrasser l'intérêt collectif. De façon générale, il affai- 
blit les capacités de transformation du Forum en un atelier hégémonique qui puisse transcender le mythe social des réunions annuelles de Davos.

\section{Conclusions}

Cet article s'est attaché à analyser le pouvoir informel à partir du cas particulier des plates-formes qui rassemblent, à l'écart des pouvoirs constitués, les sphères dirigeantes de la planète. À bien des égards, le Forum économique mondial apparaît comme l'archétype le plus exclusif et le plus puissant des clubs d'élites transnationales. Pour éviter les pièges d'une conception béhavioriste du pouvoir expliquant une décision politique formelle à partir d'une position tenue dans un club par définition fermé, cette contribution part d'une double hypothèse. Les clubs d'élites transnationales réunissent des personnalités qui occupent de facto une position de pouvoir; le principe d'exclusion qui guide ce critère d'appartenance fait aussi bien la force que la faiblesse de leur pouvoir informel.

Tant le principe d'organisation du Forum économique mondial que les modalités concrètes dans lesquelles se déroulent ses activités laissent apparaître les limites du pouvoir informel exercé par ce type d'acteur non étatique sur la scène internationale. Le gigantisme des réunions de Davos restreint considérablement les possibilités d'accès exclusif et non discriminatoire au réseau célébré pour être le plus dense du monde. Cette densité, ainsi que la centralité et le confinement du réseau, constituent par ailleurs une cible privilégiée de contestation. Le problème d'image associé au décor de forteresse de Davos s'est traduit par une désaffection d'un nombre croissant de personnalités émanant aussi bien du monde des affaires que des hautes sphères de l'État. Les bases financières du WEF - fort robustes pendant longtemps - s'en trouvent fragilisées. Les bouchées doubles que les dirigeants du Forum mettent pour garantir en toute sécurité l'atmosphère de club à l'abri du monde extérieur suscitent aujourd'hui un vif débat sur le coût social et financier endossé à cet égard par les pouvoirs publics helvétiques. Enfin, la réorientation stratégique du Forum vers des ateliers hégémoniques se situant à un niveau supérieur d'institutionnalisation semble semée d'embûches: elle continue de dépendre du succès et de la réputation des messes de Davos; elle peine à inclure un ensemble suffisamment large d'interlocuteurs stratégiques parmi les groupes d'opposition; et elle repose principalement sur une catégorie de dirigeants dont l'expertise managériale n'a d'égale que le manque de sens de l'engagement transcendant leur intérêt individuel.

Ainsi, sans nier les capacités évidentes d'influence d'un nombre important d'individus associés aux activités du Forum économique mondial, cette contribution identifie 
un ensemble de problèmes qui relativisent son pouvoir informel. Trois conclusions peuvent être dégagées à partir de la démarche proposée dans ce travail. Tout d'abord, la théorie sorélienne du mythe social offre une matrice d'analyse qui permet de clarifier un angle mort des travaux d'économie politique internationale consacrés aux transformations actuelles de la scène mondiale. En procédant au décloisonnement de la souveraineté des États dans l'ensemble plus large des relations qui structurent les sociétés capitalistes, ces travaux s'appuient sur une analyse de classes pour mettre en lumière les agents de la mondialisation. La plupart d'entre eux restent toutefois laconiques sur les processus de socialisation qui ont lieu dans un tel contexte. Avec le concept de mythe social, on se donne les moyens de spécifier les conditions dans lesquelles se renforce une conscience de classe: l'engagement unique d'individus dans une action collective d'envergure sépare clairement les groupes sociaux. La mobilisation tire sa force du clivage qui clarifie et, par conséquent, contribue à mieux fixer la manière dont les personnes s'identifient entre elles et projettent leur action dans le futur. Comme l'explique Cox, «le contenu du mythe est moins important que son pouvoir mobilisateur pour clarifier les points de discorde et stimuler l'action en faveur du changement ${ }^{67}$. N'est-ce pas là la quintessence du message supposant l'existence d'un "esprit de Davos»? Or, comme nous l'avons vu, Sorel reconnaissait qu'une telle dynamique ne pouvait pas s'extraire d'un processus institutionnel plus en profondeur.

Cela nous amène au second point: la question du pouvoir. De nombreux chercheurs se sont aujourd'hui approprié le concept gramscien d'hégémonie pour appréhender les multiples facettes du pouvoir structurel à l'échelle mondiale. Or, sur ce point, les écrits de Gramsci sont pour ainsi dire inexistants. Une transposition de ses réflexions sur le plan international nécessite un important travail de réinterprétation, de redéfinition, qui a pour corollaire le dépassement des catégories originelles ${ }^{68}$. Il manque notamment une analyse précise des mécanismes de l'hégémonie, des conditions concrètes qui président à la mise en œuvre d'un ordre à l'échelle du monde et non pas d'un État souverain. Ici encore, les réflexions de Sorel sur le mythe social conduisent à une innovation conceptuelle qui souligne la polarité de la dynamique sociale complexe dans laquelle se met en œuvre le pouvoir hégémonique. C'est à ce titre que mythes sociaux et direction hégémonique s'apparentent aux deux bornes d'un continuum entre, d'une part, la vanité

${ }_{7}$ Cox, Robert W., op. cit., p. 29.

${ }^{68}$ Randall Germain and Michael Kenny, «Engaging Gramsci: International Theory and the New Gramscians", Review of International Studies, Vol. 24, $\mathrm{N}^{\circ} 1,1998$, pp. 3-21. d'individus surestimant leur capacité à transformer à eux seuls le monde, et de l'autre, le contrôle des institutions publiques par une configuration de forces particulière.

Le troisième point a spécifiquement trait au pouvoir informel. Les différents canaux institutionnels par les- 
quels les acteurs non étatiques sont en mesure d'influencer l'architecture normative internationale font aujourd'hui l'objet d'un nombre croissant de travaux consacrés à la mondialisation. Or, comme le notent Higgot et ses coauteurs, «le partage de l'autorité entre les différents acteurs concernés varie selon l'analyse de la mondialisation retenue» ${ }^{69}$. Dans cette optique, la nouvelle emprise des acteurs non étatiques sur la scène internationale doit être pensée au-delà de son évidence empirique. Par ses réflexions sur ce qui fait tant la force que la faiblesse des mobilisations sociales coupées des institutions légitimes de l'espace public, Sorel ouvre une piste de recherches originale pour élaborer ce que j'ai nommé une herméneutique des limites. Cette approche vise à expliciter les limites du pouvoir conféré à - ou capté par - la catégorie des acteurs non étatiques sur le plan international. Face à l'impasse d'une recherche orientée vers des preuves tangibles de pouvoir, cette démarche prend acte du périmètre restreint dans lequel s'exerce l'influence par définition informelle et exclusive de tels acteurs. Une herméneutique des limites permet ainsi de circonscrire et, partant, relativiser le pouvoir auquel concluent la plupart des travaux. Les clubs d'élites transnationales représentent un cas exemplaire de cette problématique. Quelles que soient les hiérarchies qu'ils reproduisent à l'abri de portes closes, ils rassemblent des individus qui sont de facto en position de pouvoir. Certes, les clubs d'élites constituent des plates-formes informelles précieuses pour mobiliser un réseau et conduire en toute discrétion des négociations. Ils offrent aussi le support requis pour les personnalités impliquées dans une forme ou une autre de planification stratégique de l'avenir à long terme du capitalisme. Mais leur rôle dans l'espace public bute contre les limites intrinsèques de leur principe d'organisation. Coupés de la société et sans délégation formelle d'autorité à leur égard, plus leur influence sera grande, plus manifeste deviendra le déficit de légitimité du pouvoir informel et privé qu'ils incarnent. La faiblesse de leur ambition à forger le débat public tient dans ce paradoxe. Tôt ou tard, une telle situation favorise le développement de forces sociales contestant l'existence même des clubs d'élites transnationales. 